\title{
Sex Moderates the Effects of the Sorll Gene rs2070045 Polymorphism on Cognitive Impairment and Disruption of the Cingulum Integrity in Healthy Elderly
}

\author{
Ying Liang ${ }^{1,2,6}, \mathrm{He} \mathrm{Li}^{2,3,6}$, Chenlong Lv ${ }^{2,4,6}$, Ni Shu ${ }^{1,2}$, Kewei Chen ${ }^{5}, \mathrm{Xin} \mathrm{Li}^{1,2}$, Junying Zhang ${ }^{1,2}$, Liangping Hu${ }^{4}$ \\ and Zhanjun Zhang*, 1,2 \\ 'State Key Laboratory of Cognitive Neuroscience and Learning and IDG/McGovern Institute for Brain Research, Beijing Normal University, Beijing, \\ China; ${ }^{2}$ BABRI Centre, Beijing Normal University, Beijing, China; ${ }^{3}$ Institute of Basic Research in Clinical Medicine, China Academy of Chinese \\ Medical Sciences, Beijing, China; ${ }^{4}$ Consulting Center of Biomedical Statistics, Academy of Military Medical Sciences, Beijing, People's Republic of \\ China; ${ }^{5}$ Computational Image Analysis Banner Alzheimer's Institute, Phoenix, AZ, USA
}

The SORLI rs2070045 polymorphism was reported to be associated with SorLA expression in the brain and the risk of late-onset Alzheimer's disease (AD). However, the influence of this polymorphism on cognitive functioning is likely to be moderated by sex. This study aimed to examine the sex moderation on the effects of rs2070045 on neuropsychological performance and the cingulum integrity in Chinese Han population. In this study, 780 non-demented older adults completed a battery of neuropsychological scales. Diffusion tensor images (DTI) of 126 subjects were acquired. We adopted the atlas-based segmentation strategy for calculating the DTI indices of the bilateral cingulum and cingulum hippocampal part for each subject. We used a multivariate analysis of variance (MANOVA) to compare the cognitive performance and DTI differences between the rs2070045 genotype. Controlling for age, education, and the APOE $\varepsilon 4$ status, the influence of sex on the effects of the rs 2070045 polymorphism on executive function was observed. We also found an interaction between sex and the rs 2070045 polymorphism on the white matter (WM) microstructure of the left cingulum hippocampal part. Furthermore, the mean diffusivity and axial diffusivity of the tract were associated with Trail Making Test performance in $T / T$ men. These results hint that sex moderates the association between the rs 2070045 polymorphism and executive function, as well as the WM integrity of the left cingulum hippocampal part. Our findings underscore the importance of considering the influence of sex when examining the candidate genes for cognitive abilities and AD.

Neuropsychopharmacology (20I5) 40, I519-1527; doi:I0.1038/npp.20I5.I; published online 4 February 2015

\section{INTRODUCTION}

It is well-accepted that in addition to environmental factors, genetic factors have impact on cognitive aging and dementia. For example, the apolipoprotein E (APOE) 84 allele is the best-established genetic risk factor for Alzheimer's disease (AD), which significantly increases the incidence of lateonset $\mathrm{AD}$ and decreases the mean onset age of $\mathrm{AD}$ (Corder et al, 1993). The APOE $\varepsilon 4$ allele is also related to cognitive impairment and greater cognitive decline in non-demented elderly (Bretsky et al, 2003; Small et al, 2004). In addition to the $A P O E$ gene, several other genes have been identified to be associated with $\mathrm{AD}$ risk (Tanzi and Bertram, 2005; Bertram et al, 2007). The neuronal sortilin-related receptor (SORL1) gene, which has a key role in the differential

*Correspondence: Dr Z Zhang, BABRI Centre, State Key Laboratory of Cognitive Neuroscience and Learning, Beijing Normal University, Beijing 100875, China, Tel: +86 1058802005, Fax: +86 1058802005, E-mail: zhang_rzs@bnu.edu.cn

${ }^{6}$ These authors contributed equally to the manuscript. Received 6 November 20 14; revised 13 December 20 I4; accepted 29 December 2014; accepted article preview online 19 January 20 I5; sorting of the amyloid precursor protein (APP) and regulating the amyloid- $\beta$ ( $\mathrm{A} \beta$ ) production (Offe et al, 2006; Rogaeva et al, 2007), is one of the most studied putative Alzheimer's disease (AD) susceptibility genes (Lee et al, 2007; Meng et al, 2007; Kolsch et al, 2009).

The synonymous single-nucleotide polymorphism (SNP) rs2070045 is located in the $3^{\prime}$ region of the SORL1 gene. The SNP genotype alters codon usage for serine in the risk variant, suggesting reduced translation efficiency as the molecular basis of insufficient sorting protein-related receptor with A-type repeats (SorLA) expression (Zeeberg, 2002; Caglayan et al, 2012). A brain autopsy study demonstrated the correlation of a SORL1 haplotype that consists of rs2070045 to SorLA expression in the brain of AD patients (Caglayan et al, 2012). The SNP rs2070045 and haplotypes that encompass this SNP have been described to be associated with the risk of late-onset $\mathrm{AD}$ in various ethnic populations (Rogaeva et al, 2007; Tan et al, 2009; Ning et al, 2010; Reitz et al, 2011; Xue et al, 2014). The rs2070045 G allele has been recognized as a risk factor for $\mathrm{AD}$ in populations that are mainly of Caucasian origin (Rogaeva et al, 2007; Reitz et al, 2011). However, a recent study 
showed that the $\mathrm{T}$ allele of $\mathrm{rs} 2070045$ in the SORL1 was significantly associated with increased $\mathrm{AD}$ risk in the Chinese Han population (Xue et al, 2014). These results suggest that the effects of the rs2070045 polymorphism might not be consistent among the different ethnic groups.

Regarding the relationship between the variants in the SORL1 gene and cognitive abilities, both positive and negative findings have been reported (Seshadri et al, 2007; Houlihan et al, 2009; Liu et al, 2009; Shulman et al, 2010). Sex moderation of SORL1 effects on cognitive abilities could be a source of these discrepant findings. A recent large longitudinal investigation observed sex-moderated associations between the rs2070045 polymorphism and spatial, episodic memory, and verbal trajectories. Specifically, possible protective effects of the rs2070045 rare homozygote (G/G) for men's performance at least before age 75 and detrimental effects for women's performance were both shown (Reynolds et al, 2013). Moreover, Cellini et al (2009) found an association between SORL1 variants with sex in Italian patients and lateonset $\mathrm{AD}$ and suggested that the SORL1 could possibly affect $\mathrm{AD}$ risks through a female-specific mechanism.

Traditionally, the connection between the micro genotypes and the macro cognitive phenotypes is difficult to establish, and the results of related studies are often irreproducible. The recent introduction and development of imaging genetics, which constructs neuroimaging biomarkers as intermediate phenotypes for gene discovery, would improve the power of genetic association studies and bridge the gap from DNA sequences to human behavior (Meyer-Lindenberg and Weinberger, 2006).

As an important white matter (WM) tract for normal aging and AD development (Yoon et al, 2008; Catheline et al, 2010), the cingulum bundle is the major median associative WM fasciculus that connects the cingulate subregions and projects to the entorhinal cortex, having a key role in various cognitive abilities (Nestor et al, 2007; Schermuly et al, 2010). Relative to normal controls, AD patients showed lower anisotropy of cingulum bundles (Xie et al, 2005). Greater reduction of the cingulum integrity has also been observed in carriers of the APOE \&4 allele compared with non-carriers (Heise et al, 2011). It has been demonstrated that SORL1 induced widespread neuronal expression reduction in the posterior cingulate cortex and influenced the microstructure of fronto-temporal WM tracts with known susceptibility in patients with $\mathrm{AD}$, including the cingulum bundle (Liang et al, 2008; Felsky et al, 2013).

On the basis of these reported findings, we hypothesized that the effects of the SORL1 rs2070045 polymorphism on cognitive functioning and WM microstructure are moderated by sex. In addition to further confirming the effects of the SORL1 variants on cognitive function in the nondemented elderly Chinese Han population, this study aimed to examine whether sex moderates the association between the rs2070045 polymorphism and neuropsychological performance, as well as the cingulum integrity.

\section{MATERIALS AND METHODS}

\section{Participants}

Subjects were recruited from the Beijing Aging Brain Rejuvenation Initiative Study Group (BABRI), which is a longitudinal study investigating aging and cognitive impairment in urban elderly individuals in Beijing, China. There were 780 participants in this study, and all of the participants had the following properties: scored no less than 24 on the Mini-Mental-Status Examination-Chinese version (MMSE) (Zhang et al, 1990); right-handed and native Chinese speakers; 50 to 80 years of age; no less than 6 years of education; had no structural abnormalities; had no history of addiction, neurologic or psychiatric diseases; had no conditions known to influence cerebral function; and had no large vessel disease. Demographic information for each group was presented in Table 1. The study was approved by the Institutional Review Board of the Beijing Normal University Imaging Center for Brain Research. Written informed consent was obtained from each participant.

\section{Cognitive Assessment}

All participants underwent a battery of neuropsychological tests to assess cognitive abilities. General mental status was assessed with the MMSE. Episodic memory tests included the Auditory Verbal Learning Test (AVLT) (Guo et al, 2009) and Recall component of Rey-Osterrieth Complex Figure Test (ROCF) (Zhou et al, 2006). Visual-spatial ability was assessed using the Copy component of ROCF (Zhou et al, 2006) and Clock-Drawing Test (CDT) (Ishiai et al, 1993). Language ability was assessed using the Boston Naming Test (BNT) (Guo et al, 2006) and Category Verbal Fluency Test (CVFT) (Mok et al, 2004). Processing speed was assessed using the Symbol Digit Modalities Test (SDMT) (Sheridan et al, 2006) and Trail Making Test A (TMT-A) (Lu et al, 2006). Finally, executive function was assessed using the Trail Making Test B (TMT-B) (Lu et al, 2006), the Trail Making Test BA (TMT-BA) (Lu et al, 2006) and Stroop Color-word Test (Stroop) (Guo et al, 2005). Neuropsychological characterizations for each group are presented in Table 1 .

\section{Genetic Analysis}

The participants were prescreened for the rs2070045 genotype using the Custom Taqman SNP Genotyping Assays (Applied Biosystems, Foster City, CA). DNA was extracted from the blood samples of the subjects according to standard procedures to conduct a PCR for characterizing the rs2070045 genotype. An additional two SNPs, rs429358 and rs7412, which collectively form the APOE $\varepsilon 2$ (with the haplotype of rs429358-rs7412: T/T), $\varepsilon 3$ (G/T), and $\varepsilon 4$ alleles $(\mathrm{G} / \mathrm{G})$, were also genotyped. The sample success rates for all three SNPs were $100 \%$, and the reproducibility of all of the genotyping was $100 \%$ according to a duplication of at least $10 \%$ of the genotypes. According to the rs2070045 genotyping, all of the subjects were divided into three groups: $234 \mathrm{G} / \mathrm{G}, 376 \mathrm{G} / \mathrm{T}$, and $170 \mathrm{~T} / \mathrm{T}$.

\section{Image Acquisition}

Diffusion tensor images of 126 subjects were acquired using a single-shot echo-planar imaging sequence on a Siemens Trio 3.0 Tesla scanner in the Imaging Center for Brain Research at Beijing Normal University (acquisition parameters: 70 axial sections; section thickness, $2 \mathrm{~mm}$; no 
Table I Demographic and Cognitive Characteristics of all Participants

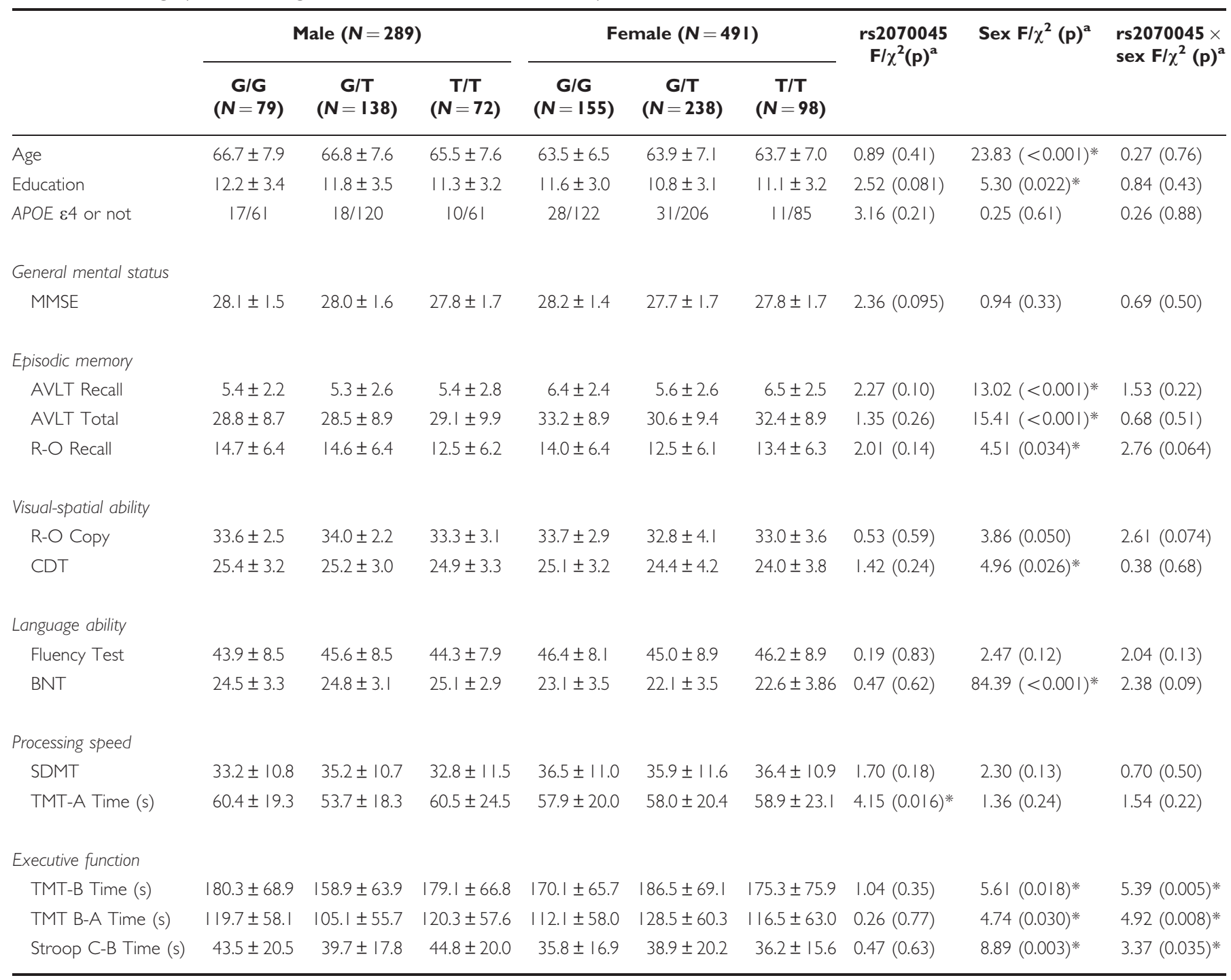

Abbreviations: AVLT, Auditory Verbal Learning Test; BNT, Boston Naming Test; CDT, Clock-Drawing Test; MMSE, Mini-Mental State Examination;

R-O, Rey-Osterrrieth Complex Figure; SDMT, Symbol Digit Modalities Test; TMT, Trail Making Test. Note: data expressed as mean \pm s.d. *Significant at $P<0.05$.

${ }^{a}$ Comparisons between groups were performed using Wald $\chi^{2}$-test for gender and APOE $\varepsilon 4$. Multivariate analysis of variance (MANOVA) was used to determine the main effect of the genotype and sex on the neuropsychological tests, as well as the interaction between them (age, education, and APOE\&4 as covariates).

section gap; 30 diffusion directions with a $b$ value of $1000 \mathrm{~s} /$ $\mathrm{mm}^{2}$ and an additional image with a $b$ value of $0 \mathrm{~s} / \mathrm{mm}^{2}$; field of view, $256 \times 256 \mathrm{~mm}^{2}$; acquisition matrix, $128 \times 128$; number of signals acquired, three). Further details of the small imaging sample are provided in Supplementary Information Table S1 and S2.

\section{Image Analysis}

Image preprocessing and analysis were carried out using FMRIB's Diffusion Toolbox (FDT) (FSL 4.1.4; www.fmrib. ox.ac.uk/fsl). First, we corrected head movement and eddy currents. A brain mask was then created by running the BET procedure on the B0 (no diffusion weighting) images. Last, DTIfit was used to fit the diffusion tensor model. The output of DTIfit yielded voxel-wise maps of the fractional anisotropy (FA), mean diffusivity (MD), axial diffusivity (DA), and radial diffusivity (DR). To investigate the diffusion changes in the cingulum, we adopted the atlas-based segmentation strategy. We registered each subject's FA, MD, DA, and DR maps to the JHU ICBM-DTI-81 template (cmrm.med.jhmi.edu/) and calculated the mean values of the bilateral cingulum and cingulum hippocampal part for each subject.

\section{Statistical Analysis}

Cognitive assessment. Demographic factors, including age and years of education, of both the rs 2070045 genotypes and sex were compared using a multivariate analysis of variance (MANOVA) or logistic regression. For the genotype and sex effects on the cognitive performance, 
comparisons were performed using a multivariate analysis of covariance (MANCOVA), with the age, education, and APOE $\varepsilon 4$ effects as covariates of no interest. All of the statistical analyses were performed with SPSS software. The HardyWeinberg test was performed by the software PLINK33.

DTI. For the diffusion alterations in the atlas-based tract ROIs, we performed a MANCOVA to compare the DTI indices differences between the rs2070045 genotype, sex, and interaction between the two (age, education, and APOE\&4 effects corrected). The Bonferroni correction was used to adjust for possible spurious findings due to multiple testing. Subsequent post hoc comparisons were performed using the Tukey's Test.

\section{Correlation Analyses Between White Matter Integrity and Neuropsychological Scores}

Pearson's partial correlation analyses controlling for age, education, and APOE $\varepsilon 4$ effects were also performed between the DTI indices and the neuropsychological scores to estimate the possible associations between the white matter integrity and cognitive functioning in the six genotype and sex groups separately.

\section{RESULTS}

\section{Demographic and Cognitive Characteristics}

In the genotype distribution in our samples, no deviation from the Hardy-Weinberg equilibrium was found $(P>0.05)$. In the large samples, we did not find any significant differences between the rs2070045 groups in the demographic results or in the $A P O E$ status (Table 1). In addition, there was also no significant SORL1 genotype by sex interactions in the demographic results or in the $A P O E$ status. We found significant differences between males and females in age and education. All of the subsequent analyses were adjusted for age and education, as well as $A P O E$ status. For the neuropsychological tests, the effect of the rs2070045 polymorphism was significant only in TMT-A $(P=0.016)$. There were significant interactions between sex and the rs2070045 polymorphisms in the TMT-B $(P=0.005)$, TMT-BA $(P=0.008)$ and Stroop C-B $(P=0.035)$ (Table 1).

\section{The Interaction Between Sex and the rs2070045 Genotype on White Matter Tracts}

Sex $\times$ rs2070045 showed a significant effect on the MD $(\mathrm{F}=5.089, P=0.008)$ and $\mathrm{DA}(\mathrm{F}=5.979, P=0.003)$ of the left cingulum hippocampal part. Especially, in males, the post-hoc results indicated that the rs2070045 G/G carriers had significantly higher $\mathrm{MD}(P=0.007)$ and $\mathrm{DA}(P=0.002)$ than the G/T carriers in the left cingulum hippocampal part (Figure 1, Table 2).

\section{Correlations Between DTI Indices and Cognitive Performance}

Only T/T men showed significant correlations between the DTI indices and neuropsychological scores. We found a significantly positive correlation between the MD values of the left cingulum hippocampal part and the Trail Making Test performance in T/T men (TMT-B: $r=0.750, P=0.002$; TMT-BA: $r=0.715, P=0.004)$. In the T/T men group, the DA of the left cingulum hippocampal part also showed a significantly positive correlation with the Trail Making Test performance (TMT-B: $\quad r=0.692, \quad P=0.006$; $\quad$ TMT-BA: $r=0.677, P=0.008$ ) (Figure 2).

\section{DISCUSSION}

The lipoprotein receptor SorLA has been demonstrated to link to $\mathrm{AD}$ pathologically based on its region-specific reduction in vulnerable regions of $\mathrm{AD}$ brains, and its potent effects on A $\beta$ production (Offe et al, 2006). Evidence showed that inherited or acquired changes in SorLA expression or function are mechanistically involved in causing Alzheimer's disease (Rogaeva et al, 2007). Therefore, the SORL1 gene encoding SorLA has received attention as a major genetic factor for AD. In this study, sex-moderated association between variants in the SORL1 rs2070045 and executive function performance was observed in nondemented Chinese older adults. We further substantiated the linkage between this genetic risk and cognitive function by demonstrating that the relation of the rs2070045 polymorphism to WM integrity of the left cingulum hippocampal part was also dependent on sex and that the correlations between executive function performance and the DTI indices of the left cingulum hippocampal part were significant in male $\mathrm{T} / \mathrm{T}$ carriers.

Sex influences the susceptibility to $\mathrm{AD}$, which could be partly due to the sex-specific associations between genetic factors and AD-related pathology (Cahill, 2006). For example, Cellini et al (2009) found an association between SORL1 variants and sex in patients with $\mathrm{AD}$. Their data suggest that SORL1 could affect AD through a femalespecific mechanism . By the same token, it appears that the relationships of genes or gene products to cognitive abilities also differ between the sexes in normal individuals. The interaction between sex and SORL1 on cognitive functioning was first reported by Reynolds et al (2013). They found that in men homozygous for the rs2070045 risk allele (G/G) initially showed a performance advantage over T/T and G/T carriers in verbal, spatial and memory tests, but their advantage diminished with faster rates of decline. For women subjects, G/G and G/T carriers tended to show lower performance across age compared with $\mathrm{T} / \mathrm{T}$ carriers. Our current study also observed sex moderation on the effects of SNP rs2070045 on cognitive functioning, but with a different trend: after controlling for age, education, and the APOE \&4 status, male homozygous for the $\mathrm{G}$ allele and $\mathrm{T}$ allele both performed worse than male heterozygous $\mathrm{G} / \mathrm{T}$ in executive function tests; female T-allele carriers exhibited lower performance than female G/G carriers. This discrepancy could arise from multiple factors such as study designs, cognitive parameters, sample sizes, and even ethnic background. It has been reported that the $\mathrm{G}$ allele, which is the minor allele of the rs2070045 for Caucasians and other populations, is a risk factor for AD (Rogaeva et al, 2007; Reitz et al, 2011; Reynolds et al, 2013). In this study, we found that the $\mathrm{T}$ allele is the minor allele, which is 
a
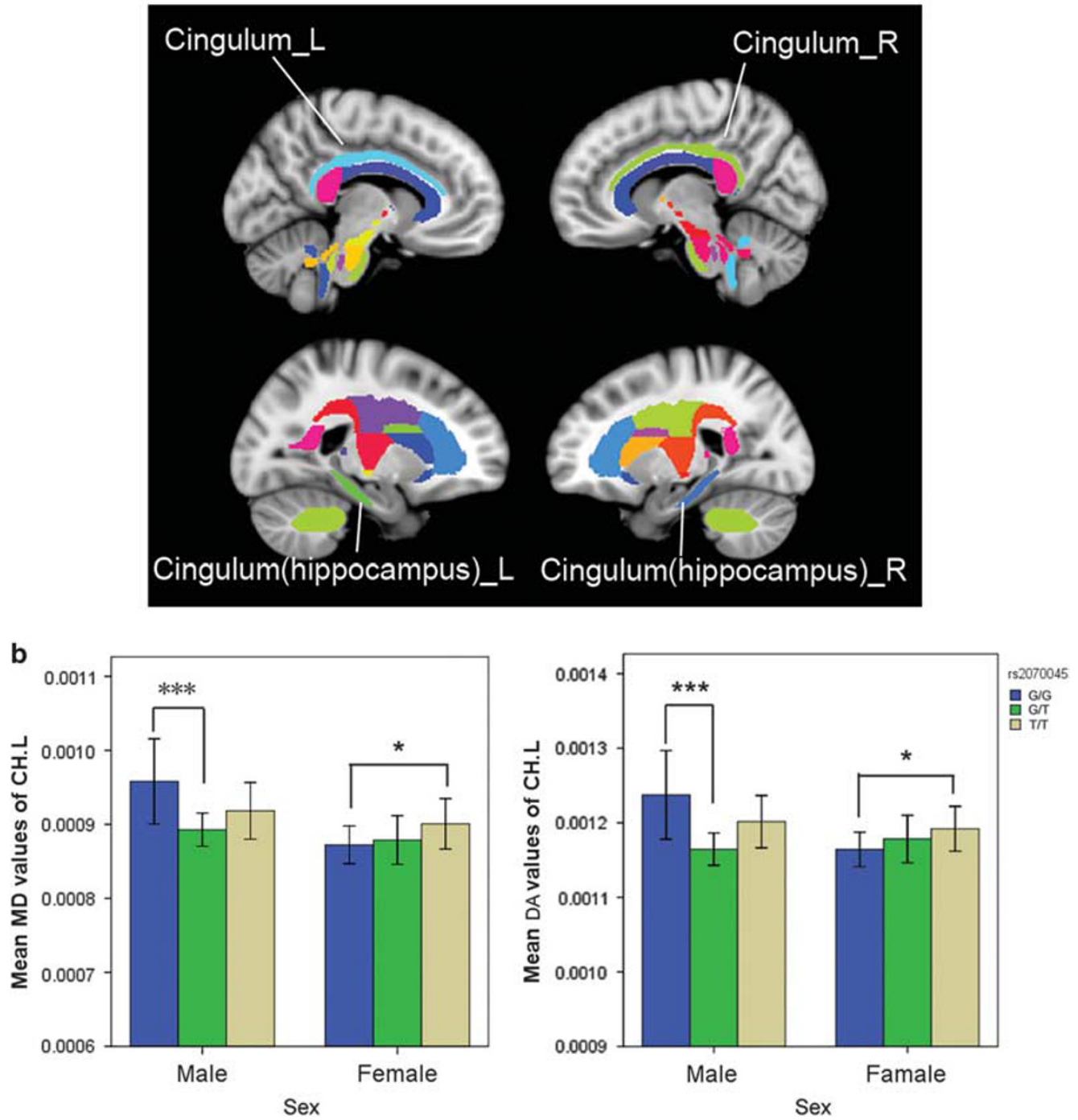

Figure I Mean diffusion metrics of the atlas-based tracts in the gene and sex groups. (a) The JHU white matter atlas in the ICBM-DTI-8I space. Colored regions indicate the bilateral cingulum and cingulum hippocampal part. (b) Post-hoc differences in the mean diffusion metrics of the atlas-based tracts among groups. $* P<0.05$ uncorrected. $* * * * P<0.05$ in Tukey's test. DA, axial diffusivity; MD, mean diffusivity.

consistent with the findings from previous studies with Chinese Han subjects (Tan et al, 2009; Ning et al, 2010; Xue et al, 2014). Recent evidence has indicated that the $\mathrm{T}$ allele of the rs 2070045 is associated with AD risk in the Chinese Han population (Xue et al, 2014). The differences in the allele and genotype frequencies as well as in the potential risk allele might cause inconsistent results between our study and study by Reynolds et al (2013). More interestingly, we observed a heterozygote advantage for the rs2070045 polymorphism in executive function. The condition in which heterozygotes show better functioning than homozygotes for quantitative traits is called positive heterosis. Positive heterosis is common in humans and has been reported for the influence on cognitive function of the catechol O-methyl transferase (COMT) and methylenetetrahydrofolate reductase (MTHFR) genetic polymorphisms (Gosso et al, 2008; Tsai et al, 2011). One possible explanation for this heterozygote advantage for the rs2070045 polymorphism is that the SNP could have both detrimental and beneficial effects on the cognitive function of elderly subjects who carry the $\mathrm{T}$ allele. Female $\mathrm{T}$ carriers exhibit poorer cognitive functioning than G/G carriers. In contrast, the $\mathrm{T}$ allele might also exert positive effects on the aging process through other pathways, which could explain the better cognitive functioning in G/T heterozygotes than in G/G homozygotes in males. Nevertheless, this heterozygote advantage requires further confirmation in other samples, including a larger sample of men. It should be noted that this explanation still needs biochemical evidence for support.

The cingulated fiber tracts have been demonstrated to be susceptible to aging (Yoon et al, 2008), and the disruption of the posterior cingulum bundles is associated with $\mathrm{AD}$ (Catheline et al, 2010). Evidence from human postmortem brain tissue showed that the expression of SorLA protein was reduced in the posterior cingulated cortex, hippocampus, and middle temporal gyrus in $\mathrm{AD}$ brains compared with control brains (Liang et al, 2008). Diffusion imaging 
Table 2 Group Comparisons of Mean Diffusion Metrix of Each ROI

\begin{tabular}{|c|c|c|c|c|c|c|c|c|c|}
\hline \multirow{2}{*}{$\begin{array}{l}\text { White matter } \\
\text { tract }\end{array}$} & \multicolumn{3}{|c|}{ Male } & \multicolumn{3}{|c|}{ Female } & \multirow[t]{2}{*}{ rs2070045 F(p $)^{a}$} & \multirow[t]{2}{*}{$\operatorname{sex} F(p)^{a}$} & \multirow{2}{*}{$\begin{array}{l}\text { rs2070045 } \times \\
\operatorname{sex} F(p)^{a}\end{array}$} \\
\hline & $\begin{array}{c}\mathbf{G} / \mathbf{G} \\
(N=19)\end{array}$ & $\begin{array}{c}\mathbf{G} / \mathbf{T} \\
(N=24)\end{array}$ & $\begin{array}{c}T / T \\
(N=18)\end{array}$ & $\begin{array}{c}\mathbf{G} / \mathbf{G} \\
(N=24)\end{array}$ & $\begin{array}{c}\mathbf{G} / \mathbf{T} \\
(N=25)\end{array}$ & $\begin{array}{c}T / T \\
(N=16)\end{array}$ & & & \\
\hline \multicolumn{10}{|l|}{$F A$} \\
\hline Cingulum_R. & $0.36 \pm 0.03$ & $0.37 \pm 0.02$ & $0.35 \pm 0.03$ & $0.37 \pm 0.03$ & $0.37 \pm 0.03$ & $0.37 \pm 0.03$ & $0.39(0.68)$ & $2.85(0.094)$ & $0.39(0.68)$ \\
\hline Cingulum_l. & $0.37 \pm 0.03$ & $0.37 \pm 0.02$ & $0.36 \pm 0.02$ & $0.37 \pm 0.03$ & $0.38 \pm 0.03$ & $0.38 \pm 0.03$ & $0.31(0.74)$ & $3.75(0.055)$ & $0.65(0.53)$ \\
\hline \multicolumn{10}{|l|}{$M D\left(\times 10^{-4}\right)$} \\
\hline Cingulum_R. & $7.78 \pm 0.36$ & $7.70 \pm 0.36$ & $7.90 \pm 0.39$ & $7.67 \pm 0.35$ & $7.80 \pm 0.32$ & $7.74 \pm 0.23$ & $1.57(0.21)$ & $0.24(0.62)$ & $3.02(0.053)$ \\
\hline Cingulum_I. & $7.96 \pm 0.28$ & $7.93 \pm 0.30$ & $8.05 \pm 0.23$ & $7.85 \pm 0.39$ & $7.93 \pm 0.30$ & $7.97 \pm 0.28$ & $2.42(0.09)$ & $0.00(1.00)$ & $1.26(0.29)$ \\
\hline Cingulum_hippocampus_R. & $9.19 \pm 0.91$ & $8.75 \pm 0.64$ & $8.99 \pm 0.77$ & $8.68 \pm 0.66$ & $8.56 \pm 0.56$ & $8.55 \pm 0.50$ & $2.00(0.14)$ & $3.61(0.060)$ & $1.17(0.32)$ \\
\hline Cingulum_l. & $11.34 \pm 0.29$ & $11.28 \pm 0.38$ & $11.34 \pm 0.37$ & $11.22 \pm 0.36$ & $11.38 \pm 0.33$ & $11.45 \pm 0.34$ & $1.43(0.25)$ & $1.72(0.19)$ & $2.40(0.096)$ \\
\hline Cingulum_hippocampus_R. & $12.07 \pm 0.93$ & $11.53 \pm 0.59$ & $11.84 \pm 0.80$ & $11.63 \pm 0.62$ & $|1.63 \pm 0.5|$ & $11.53 \pm 0.49$ & $2.00(0.14)$ & $0.32(0.57)$ & $2.67(0.07)$ \\
\hline Cingulum_ hippocampus_I. & $12.38 \pm 1.23$ & $|1.65 \pm 0.5|$ & $12.02 \pm 0.70$ & $11.63 \pm 0.56$ & $11.78 \pm 0.77$ & $11.92 \pm 0.56$ & $2.66(0.075)$ & $0.12(0.73)$ & $5.98(0.003)^{*}$ \\
\hline \multicolumn{10}{|l|}{$D R\left(\times 10^{-4}\right)$} \\
\hline Cingulum_R. & $6.23 \pm 0.41$ & $6.14 \pm 0.41$ & $6.35 \pm 0.46$ & $6.11 \pm 0.43$ & $6.19 \pm 0.36$ & $6.14 \pm 0.31$ & $0.59(0.56)$ & $1.12(0.29)$ & $0.76(0.47)$ \\
\hline Cingulum_l. & $6.27 \pm 0.36$ & $6.27 \pm 0.36$ & $6.39 \pm 0.26$ & $6.18 \pm 0.46$ & $6.21 \pm 0.36$ & $6.24 \pm 0.37$ & $0.69(0.50)$ & $2.20(0.14)$ & $0.093(0.9 \mid)$ \\
\hline Cingulum_ hippocampus_R. & $7.75 \pm 0.91$ & $7.56 \pm 1.19$ & $7.53 \pm 0.80$ & $7.19 \pm 0.71$ & $7.06 \pm 0.65$ & $7.10 \pm 0.56$ & $0.31(0.74)$ & $8.91(0.003)^{*}$ & $0.068(0.93)$ \\
\hline Cingulum_ hippocampus_l. & $8.18 \pm 1.18$ & $7.75 \pm 1.16$ & $7.73 \pm 0.86$ & $7.27 \pm 0.67$ & $7.34 \pm 0.82$ & $7.52 \pm 0.70$ & $0.30(0.74)$ & $8.76(0.004)^{*}$ & $1.28(0.28)$ \\
\hline
\end{tabular}

Abbreviations: DA, axial diffusivity; DR, radial diffusivity; FA, fractional anisotropy; MD, mean diffusivity. Note: data expressed as mean \pm SD. P-values are all raw $P$-values. $* P<0.05$ significant after Bonferroni correction.

aultivariate analysis of variance (MANOVA) was used to determine the main effect of the genotype and sex on the DTI indices, as well as the interaction between them (age, education, and APOE\&4 as covariates).

could probe white matter microstructure in vivo and indicate the underlying biophysical properties. A recent DTI study of WM microstructure in healthy Caucasian subjects reported that the SORL1 risk variants (rs689021) predicted lower FA in an age-independent manner in the cingulum bundle and several other fronto-temporal WM tracts. However, the interaction between sex and the SORL1 variants was not explored (Felsky et al, 2013). By performing analysis on a pre-defined ROI that was specially designed for detecting the whole cingulum bundle, we found the interaction between sex and the SORL1 rs2070045 polymorphism on the MD and DA value of the left cingulum hippocampal part. Increased MD was reported to be caused by decrease in membrane density and DA could reflect the axonal injury (Douaud et al, 2011; Wheeler-Kingshott and Cercignani, 2009). Specifically, G/G men showed reduced integrity of the left cingulum hippocampal part compared with G/T men, and T/T women exhibited reduced integrity compared with G/G women, which was consistent with our behavioral observations. Although not significant, there is a similar tendency in the sex differences in the association between the SORL1 variants and the integrity of the right cingulum hippocampal part. We guess that sex could regulate the relationship between the rs2070045 polymorphism and WM integrity through modulating the allelic differences in the expression of SorLA in the cingulate cortex, which lead to subtle changes in the $A \beta$ concentration.

Some previous DTI evidence suggests that the cingulum bundle could carry anterior-posterior connections that are important for executive function (O'Sullivan et al, 2005; Kantarci et al, 2011). However, the correlation analyses in these studies have not been separated by sex and by SORL1 genotypes. In this study, the MD and DA of the left cingulum hippocampal part were found to be associated with TMT performance only in males who were homozygous for the $\mathrm{T}$ allele. As shown in Figure 2, G/T men showed the best executive performance and cingulum integrity compared with the other two genotype groups, whose plots fell in the third quadrant. The opposite situation was observed in G/G men. The scatter plots of T/T men were more widely distributed than the G/T and G/ $\mathrm{G}$ groups. Further studies with larger sample sizes might be necessary to determine the relationship between the cingulum integrity and executive performance. Moreover, we did not find any significant association in the females. Our findings indicate that sex and the rs2070045 polymorphism could affect the relationship between the cingulum integrity and executive function.

There is conflicting evidence regarding the relationships between the SORL1 gene and cognitive functioning 
a
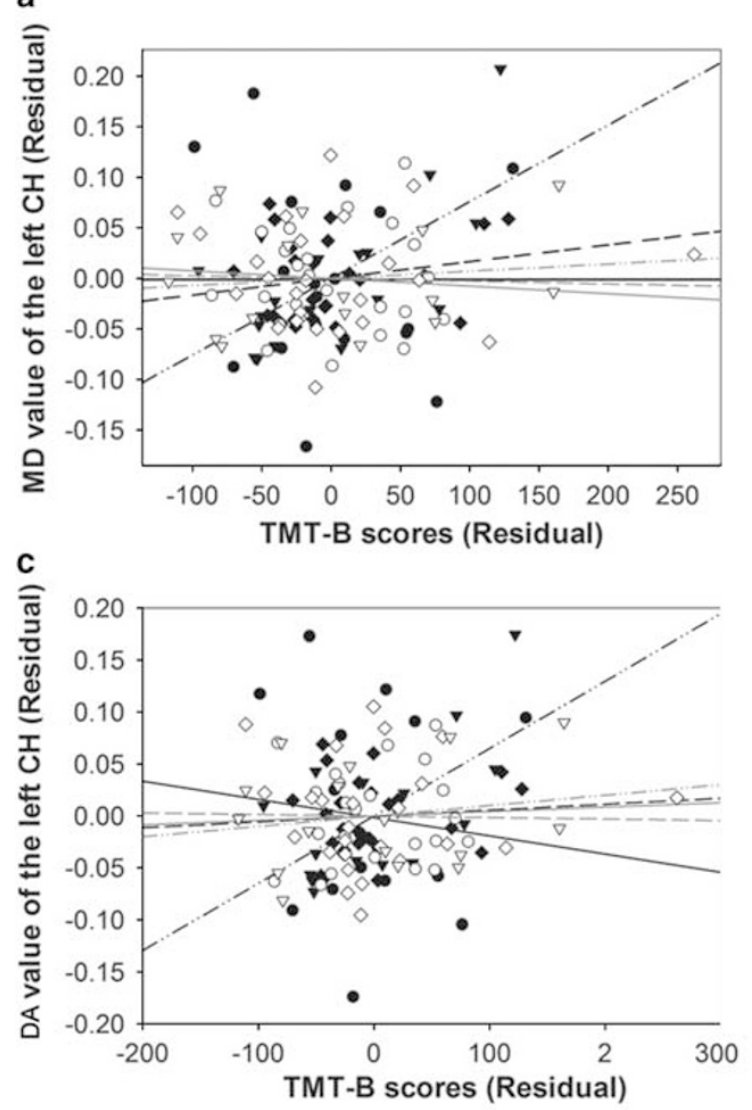

b

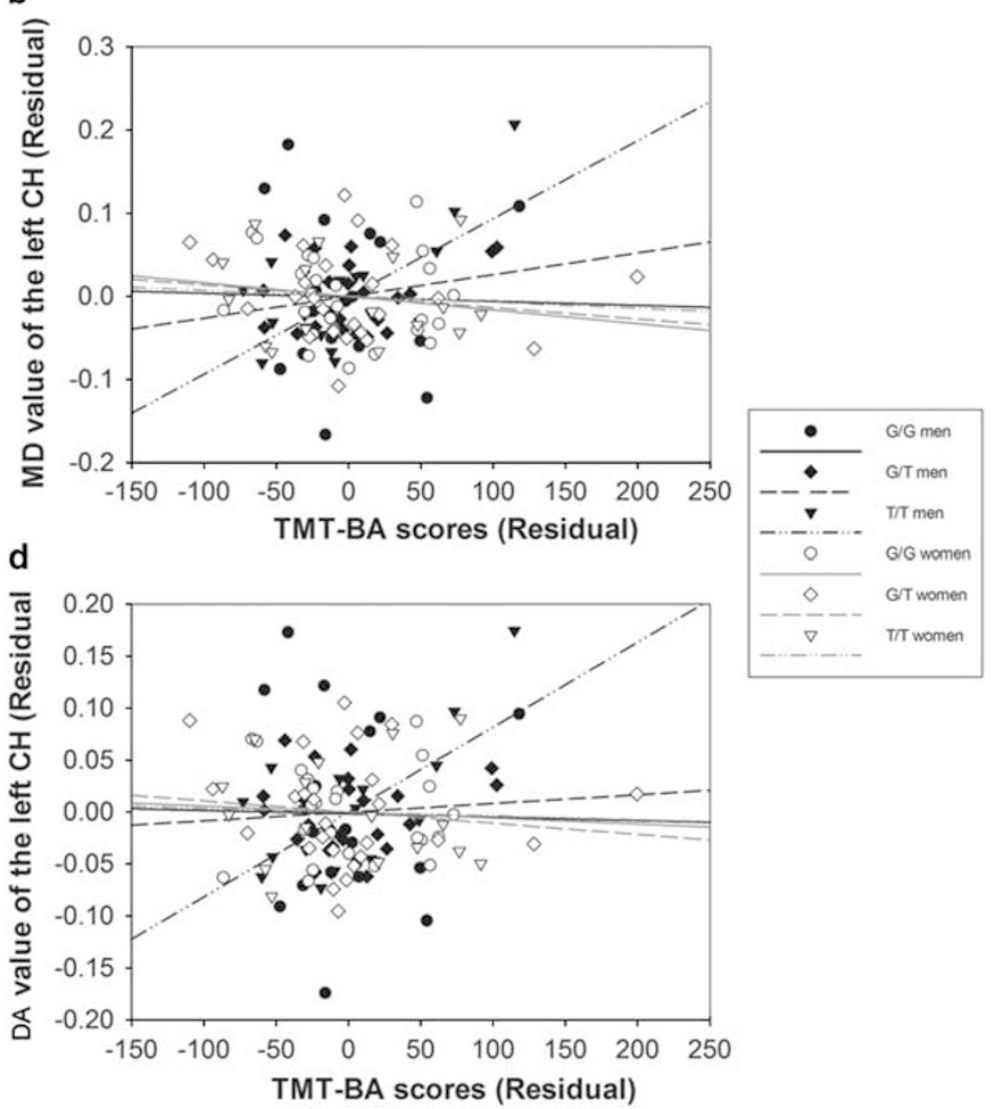

Figure 2 Correlations between the white matter and neuropsychological tests in the six genotype and sex groups, separately. (a) Plots showing the significant correlation between the MD of the left cingulum hippocampal part and Trail Making Test B (TMT-B) scores in T/T men. (b) Plots showing the significant correlation between the mean diffusivity of the left cingulum hippocampal part and TMT-BA scores in T/T men. (c) Plots showing the significant correlation between the DA of the left cingulum hippocampal part and TMT-B scores in T/T men. (d) Plots showing the significant correlation between the DA of the left cingulum hippocampal part and TMT-BA scores in T/T men. No significant correlation was found in the other group.

(Seshadri et al, 2007; Houlihan et al, 2009; Liu et al, 2009; Shulman et al, 2010), and the gene variants confer a smallto-modest risk of $\mathrm{AD}$ (Lee et al, 2008; Reitz et al, 2011). In our study, the main effect of the rs2070045 polymorphism was not significant on any of the cognitive measures except on Trail Making Test-A. Nevertheless, the significant interaction of sex with gene was observed on executive function. Thus, the sex differences should be considered when examining the effects of SORL1 on cognitive function as well as AD.

Several limitations of this study should be acknowledged. First, the male and female subjects were not matched in terms of age and education, although demographic variables have been controlled in all of the analyses. Second, similar to many other genetic association studies, we did not measure SorLA expression in the brain. Such evidence is necessary to clarify the exact mechanisms of how sex moderates the association between the SORL1 variants and cognition, as well as WM integrity. Finally, in the present study, only the rs2070045 SNP was singled out. The reason is that sex exerts the most evident moderation on this SNP (Reynolds et al, 2013). Future studies that examine other SORL1 SNPs and haplotypes could provide comprehensive descriptions of the interaction between sex and the SORL1 gene.
In summary, we observed sex-moderated association of the SORL1 rs2070045 polymorphism and executive function. The relationship of the SNP to the integrity of the left cingulum hippocampal part was also found to be dependent on sex. These results hint that sex moderation of the SORL1 effects on executive function could be related to its moderation on the SORL1 effects on the cingulum bundle. Our findings underscore the importance of integrating sex and genetic susceptibility when examining candidate genes for cognitive abilities and $\mathrm{AD}$. A prospective study with a larger sample size and a longitudinal design would permit further clarification on the extent to which the effects of SORL1 variants on cognitive aging are moderated by sex.

\section{FUNDING AND DISCLOSURE}

This study was supported by the State Key Program of National Natural Science of China (No. 81430100), the Beijing New Medical Discipline Based Group (100270569), the National Natural Science Foundation of China (Nos. 30873458, and 81173460), the Project of Institute of Basic Research in Clinical Medicine, the China Academy of Chinese Medical Sciences (Z0251), the Program for New Century Excellent Talents in University (NCET-10-0249), 
the Open Research Fund of the State Key Laboratory of Cognitive Neuroscience and Learning, and the National Institute on Aging (R01AG031581). The authors declare no conflicts of interest.

\section{Author Contributions}

Z.J.Z. had full access to all of the data in the study and takes responsibility for the integrity of the data and the accuracy of the data analysis. Z.J.Z. conceived the original idea for the study and supervised in the conception. Y.L., H.L., C.L.L., X.L., and J.Y.Z. recruited the study population and conducted the neuropsychological tests. Y.L., H.L., and C.L.L. analyzed the data. Y.L., H.L., and C.L.L. drafted the manuscript. N.S., K.W.C. and L.P.H revised the manuscript. All authors read and approved the final manuscript.

\section{REFERENCES}

Bertram L, McQueen MB, Mullin K, Blacker D, Tanzi RE (2007). Systematic meta-analyses of Alzheimer disease genetic association studies: the AlzGene database. Nat Genet 39: 17-23.

Bretsky P, Guralnik JM, Launer L, Albert M, Seeman TEMacArthur Studies of Successful A (2003). The role of APOE-epsilon4 in longitudinal cognitive decline: MacArthur Studies of Successful Aging. Neurology 60: 1077-1081.

Caglayan S, Bauerfeind A, Schmidt V, Carlo AS, Prabakaran T, Hubner $\mathrm{N}$ et al (2012). Identification of Alzheimer disease risk genotype that predicts efficiency of SORL1 expression in the brain. Arch Neurol 69: 373-379.

Cahill L (2006). Why sex matters for neuroscience. Nat Rev Neurosci 7: 477-484.

Catheline G, Periot O, Amirault M, Braun M, Dartigues JF, Auriacombe $S$ et al (2010). Distinctive alterations of the cingulum bundle during aging and Alzheimer's disease. Neurobiol Aging 31: 1582-1592.

Cellini E, Tedde A, Bagnoli S, Pradella S, Piacentini S, Sorbi S et al (2009). Implication of sex and SORL1 variants in italian patients with Alzheimer disease. Arch Neurol 66: 1260-1266.

Corder EH, Saunders AM, Strittmatter WJ, Schmechel DE, Gaskell PC, Small GW et al (1993). Gene dose of apolipoprotein E type 4 allele and the risk of Alzheimer's disease in late onset families. Science 261: 921-923.

Douaud G, Jbabdi S, Behrens TE, Menke RA, Gass A, Monsch AU et al (2011). DTI measures in crossing-fibre areas: Increased diffusion anisotropy reveals early white matter alteration in MCI and mild Alzheimer's disease. NeuroImage 55: 880-890.

Felsky D, Szeszko P, Yu L, Honer WG, De Jager PL, Schneider JA et al (2013). The SORL1 gene and convergent neural risk for Alzheimer's disease across the human lifespan. Mol Psychiatr 19: 1125-1132.

Gosso MF, de Geus EJ, Polderman TJ, Boomsma DI, Heutink P, Posthuma D (2008). Catechol O-methyl transferase and dopamine D2 receptor gene polymorphisms: evidence of positive heterosis and gene-gene interaction on working memory functioning. Eur J Hum Genet 16: 1075-1082.

Guo Q, Zhao Q, Chen M, Ding D, Hong Z (2009). A comparison study of mild cognitive impairment with 3 memory tests among Chinese individuals. Alzheimer Dis Assoc Disord 23: 253-259.

Guo QH, Hong Z, Lv CZ, Zhou Y, Lu JC, Ding D (2005). Application of Stroop color-word test on Chinese elderly patients with mild cognitive impairment and mild Alzheimer's dementia. Chinese J Neuromed 4: 701-704.

Guo QH, Hong Z, Shi WX, Sun YM, Lv CZ (2006). Boston naming test using by Chinese elderly, patient with mild cognitive impairment and Alzheimer's dementia. Chinese Mental Health J 20: 81-85.

Heise V, Filippini N, Ebmeier KP, Mackay CE (2011). The APOE varepsilon4 allele modulates brain white matter integrity in healthy adults. Mol Psychiatr 16: 908-916.

Houlihan LM, Harris SE, Luciano M, Gow AJ, Starr JM, Visscher PM et al (2009). Replication study of candidate genes for cognitive abilities: the Lothian Birth Cohort 1936. Genes Brain Behavior 8: 238-247.

Ishiai S, Sugishita M, Ichikawa T, Gono S, Watabiki S (1993). Clock-drawing test and unilateral spatial neglect. Neurology 43: 106-110.

Kantarci K, Senjem ML, Avula R, Zhang B, Samikoglu AR, Weigand SD et al (2011). Diffusion tensor imaging and cognitive function in older adults with no dementia. Neurology 77: 26-34.

Kolsch H, Jessen F, Wiltfang J, Lewczuk P, Dichgans M, Teipel SJ et al (2009). Association of SORL1 gene variants with Alzheimer's disease. Brain Res 1264: 1-6.

Lee JH, Barral S, Reitz C (2008). The neuronal sortilin-related receptor gene SORL1 and late-onset Alzheimer's disease. Curr Neurol Neurosci Rep 8: 384-391.

Lee JH, Cheng R, Schupf N, Manly J, Lantigua R, Stern Y et al (2007). The association between genetic variants in SORL1 and Alzheimer disease in an urban, multiethnic, community-based cohort. Arch Neurol 64: 501-506.

Liang WS, Dunckley T, Beach TG, Grover A, Mastroeni D, Ramsey K et al (2008). Altered neuronal gene expression in brain regions differentially affected by Alzheimer's disease: a reference data set. Physiol Genomics 33: 240-256.

Liu F, Ikram MA, Janssens AC, Schuur M, de Koning I, Isaacs A et al (2009). A study of the SORL1 gene in Alzheimer's disease and cognitive function. J Alzheimer's Dis 18: 51-64.

Lu JC, GQ C, Hong Z, Shi WX, Lv CZ (2006). Trail making test used by Chinese elderly patients with mild cognitive impairment and mild Alzheimer dementia. Chinese J Clin Psychol 4: 118-121.

Meng Y, Lee JH, Cheng R St, George-Hyslop P, Mayeux R, Farrer LA (2007). Association between SORL1 and Alzheimer's disease in a genome-wide study. Neuroreport 18: 1761-1764.

Meyer-Lindenberg A, Weinberger DR (2006). Intermediate phenotypes and genetic mechanisms of psychiatric disorders. Nat Rev Neurosci 7: 818-827.

Mok EH, Lam LC, Chiu HF (2004). Category verbal fluency test performance in chinese elderly with Alzheimer's disease. Dement Geriatr Cogn Disord 18: 120-124.

Nestor PG, Kubicki M, Spencer KM, Niznikiewicz M, McCarley RW, Shenton ME (2007). Attentional networks and cingulum bundle in chronic schizophrenia. Schizophrenia Res 90: 308-315.

Ning M, Yang Y, Zhang Z, Chen Z, Zhao T, Zhang D et al (2010). Amyloid-beta-related genes SORL1 and ACE are genetically associated with risk for late-onset Alzheimer disease in the Chinese population. Alzheimer Dis Associated Disord 24: 390-396.

O’Sullivan M, Barrick TR, Morris RG, Clark CA, Markus HS (2005). Damage within a network of white matter regions underlies executive dysfunction in CADASIL. Neurology 65: 1584-1590.

Offe K, Dodson SE, Shoemaker JT, Fritz JJ, Gearing M, Levey AI et al (2006). The lipoprotein receptor LR11 regulates amyloid beta production and amyloid precursor protein traffic in endosomal compartments. J Neurosci 26: 1596-1603.

Reitz C, Cheng R, Rogaeva E, Lee JH, Tokuhiro S, Zou F et al (2011). Meta-analysis of the association between variants in SORL1 and Alzheimer disease. Arch Neurol 68: 99-106.

Reynolds CA, Zavala C, Gatz M, Vie L, Johansson B, Malmberg B et al (2013). Sortilin receptor 1 predicts longitudinal cognitive change. Neurobiol Aging 34: 1710 e1711-1710 e1718.

Rogaeva E, Meng Y, Lee JH, Gu Y, Kawarai T, Zou F et al (2007). The neuronal sortilin-related receptor SORL1 is genetically associated with Alzheimer disease. Nat Genet 39: 168-177. 
Schermuly I, Fellgiebel A, Wagner S, Yakushev I, Stoeter P, Schmitt R et al (2010). Association between cingulum bundle structure and cognitive performance: an observational study in major depression. Eur Psychiatr 25: 355-360.

Seshadri S, DeStefano AL, Au R, Massaro JM, Beiser AS, Kelly-Hayes M et al (2007). Genetic correlates of brain aging on MRI and cognitive test measures: a genome-wide association and linkage analysis in the Framingham Study. BMC Med Genet 8: S15.

Sheridan LK, Fitzgerald HE, Adams KM, Nigg JT, Martel MM, Puttler LI et al (2006). Normative Symbol Digit Modalities Test performance in a community-based sample. Arch Clin Neuropsychol 21: 23-28.

Shulman JM, Chibnik LB, Aubin C, Schneider JA, Bennett DA, De Jager PL (2010). Intermediate phenotypes identify divergent pathways to Alzheimer's disease. PloS one 5: e11244.

Small BJ, Rosnick CB, Fratiglioni L, Backman L (2004). Apolipoprotein E and cognitive performance: a meta-analysis. Psychol Aging 19: 592-600.

Tan EK, Lee J, Chen CP, Teo YY, Zhao Y, Lee WL (2009). SORL1 haplotypes modulate risk of Alzheimer's disease in Chinese. Neurobiol Aging 30: 1048-1051.

Tanzi RE, Bertram L (2005). Twenty years of the Alzheimer's disease amyloid hypothesis: a genetic perspective. Cell 120: 545-555.

Tsai SJ, Hong CJ, Yeh HL, Liou YJ, Yang AC, Liu ME et al (2011). Heterozygote advantage of the MTHFR C677T polymorphism on specific cognitive performance in elderly Chinese males without dementia. Dement Geriatr Cogn Disord 32: 159-163.

Wheeler-Kingshott CA, Cercignani M (2009). About 'axial' and 'radial' diffusivities. Magnet Reson Med 61: 1255-1260.

Xie S, Xiao JX, Wang YH, Wu HK, Gong GL, Jiang XX (2005). Evaluation of bilateral cingulum with tractography in patients with Alzheimer's disease. Neuroreport 16: 1275-1278.

Xue X, Zhang M, Lin Y, Xu E, Jia J (2014). Association between the SORL1 rs2070045 polymorphism and late-onset Alzheimer's disease: interaction with the ApoE genotype in the Chinese Han population. Neurosci Lett 559: 94-98.

Yoon B, Shim YS, Lee KS, Shon YM, Yang DW (2008). Region-specific changes of cerebral white matter during normal aging: a diffusion-tensor analysis. Archi Gerontol Geriatr 47: 129-138.

Zeeberg B (2002). Shannon information theoretic computation of synonymous codon usage biases in coding regions of human and mouse genomes. Genome Res 12: 944-955.

Zhang MY, Katzman R, Salmon D, Jin H, Cai GJ, Wang ZY et al (1990). The prevalence of dementia and Alzheimer's disease in Shanghai, China: impact of age, gender, and education. Ann Neurol 27: 428-437.

Zhou Y, Lu JC, Guo QH, Hong Z (2006). Rey-Osterriche complex figure test used to identify mild Alzheimer's disease. Chin J Clin Neurosci 14: 501-504.

Supplementary Information accompanies the paper on the Neuropsychopharmacology website (http://www.nature.com/npp) 